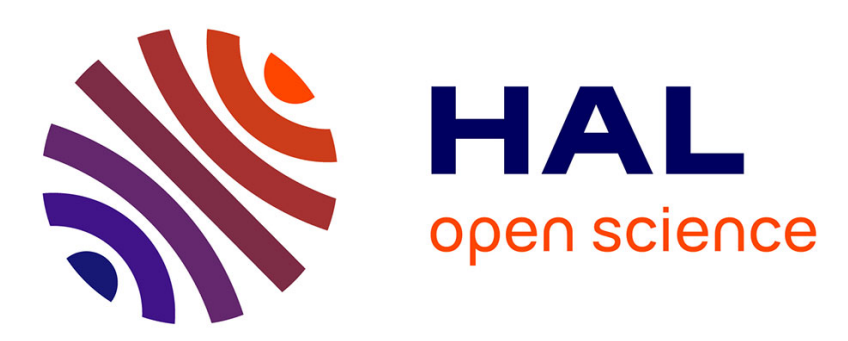

\title{
Wheat leaf bidirectional reflectance measurements: Description and quantification of the volume, specular and hot-spot scattering features
}

\author{
L Comar, B Baret, F Viénot, L Yan, B de Solan
}

\section{To cite this version:}

L Comar, B Baret, F Viénot, L Yan, B de Solan. Wheat leaf bidirectional reflectance measurements: Description and quantification of the volume, specular and hot-spot scattering features. Remote Sensing of Environment, 2012, 121, pp.26-35. 10.1016/j.rse.2011.01.028 . hal-01565506

\author{
HAL Id: hal-01565506 \\ https://hal.science/hal-01565506
}

Submitted on 8 Sep 2017

HAL is a multi-disciplinary open access archive for the deposit and dissemination of scientific research documents, whether they are published or not. The documents may come from teaching and research institutions in France or abroad, or from public or private research centers.
L'archive ouverte pluridisciplinaire HAL, est destinée au dépôt et à la diffusion de documents scientifiques de niveau recherche, publiés ou non, émanant des établissements d'enseignement et de recherche français ou étrangers, des laboratoires publics ou privés. 


\title{
Wheat leaf bidirectional reflectance measurements: Description and quantification of the volume, specular and hot-spot scattering features
}

\author{
A. Comar ${ }^{\text {a,c,*, }}$, F. Baret ${ }^{\text {a }}$, F. Viénot ${ }^{\text {b }}$, L. Yan ${ }^{\text {a }}$, B. de Solan ${ }^{\text {a,c }}$ \\ a UMR EMMAH, Inra PACA, UAPV, Domaine Saint-Paul, Site Agroparc, 84914 Avignon, France \\ b Muséum national d'histoire naturelle, CRCC (MNHN-CNRS-MCC), 36 rue Geoffroy Saint-Hilaire, 75005 Paris, France \\ c ARVALIS, Institut du végétal, 3 rue Joseph et Marie Hackin, 75116 Paris, France
}

\section{A R T I C L E I N F O}

\section{Article history:}

Received 28 July 2010

Received in revised form 14 January 2011

Accepted 14 January 2011

Available online $\mathrm{xxxx}$

\section{Keywords:}

Wheat

BRF

BRDF

Hotspot

Specular

Volume scattering

Conoscope

Fourier optics

\begin{abstract}
A B S T R A C T
This study focuses on the directionality of wheat leaf reflectance as a function of leaf surface characteristics. Wheat leaf BRF measurements were completed under $45^{\circ}$ zenith illumination angle in three visible broad spectral bands with a conoscope that provides very high angular resolution data over a large portion of the whole hemisphere, including around the illumination direction. The measurements show a clear anisotropy with a specular lobe in the forward scattering direction and a small but significant hotspot feature in the backward scattering direction. The BRF directional features further depend on the illumination orientation because of the leaf roughness created by longitudinal veins: the specular lobe was more pronounced when the illumination was perpendicular to the veins, while specular reflection was more spread over azimuths for longitudinal illumination. Moreover, a sharp hotspot feature was observed for transversal illumination where the apparent roughness is the largest. The scattering was tentatively decomposed into specular, hotspot and isotropic components. Results showed that the hotspot contribution to the directional hemispherical reflectance factor (DHRF) was marginal conversely to that of the specular component that ranges between 0.036 and 0.050 (absolute DHRF value). The specular component was almost the same in the three visible bands considered. The isotropic component originating from volume scattering was contributing the most to the DHRF and was depending on wavelength, ranging between 0.055 and 0.097 in absolute DHRF value. A simple model was proposed to estimate the volume scattering from the isotropic and the surface components. Consequences of these findings were drawn on the ability to estimate leaf biochemical composition independently from leaf surface scattering, as well as on the interpretation of remote sensing at the canopy level.
\end{abstract}

(c) 2012 Elsevier Inc. All rights reserved.

\section{Introduction}

The description of leaf optical properties, i.e. reflectance, transmittance and absorptance is important for understanding several processes intervening within the functioning of the canopy (Terashima \& Saeki, 1983). Hence, it may serve a wide range of environmental, ecological and agricultural related applications. Emphasis was mainly put on the capacity to estimate the leaf biochemical composition from the spectral variation of optical properties. This was illustrated by several studies over a range of scales, from the leaf (Fourty et al., 1996; Gitelson et al., 2003; Markwell et al., 1995), high throughput phenotyping issues (Mistele \& Schmidhalter, 2008), up to remote sensing applications (Fourty \& Baret, 1997; Fourty et al., 1996; Jacquemoud et al., 2009). However, very little attention has been paid to leaf directional properties that may induce problems in the retrieval of leaf biochemistry at the leaf and canopy levels, as well as

\footnotetext{
* Corresponding author.

E-mail address: alexis.comar@etd.univ-avignon.fr (A. Comar).
}

when exploiting the bidirectional reflectance distribution function (BRDF) to estimate canopy structural attributes such as Leaf Area Index (LAI). As a matter of fact, information on the biochemistry comes from the absorption by the constituents occurring within the leaf, whereas the anisotropy of leaf reflectance originates mainly from processes taking place at the leaf surface including specular reflection (Breece \& Holmes, 1971; Grant et al., 1987), shadowing created by leaf roughness (Bousquet et al., 2005) and scattering by trichomes on leaves (Holmes \& Keiller, 2002; Levizou et al., 2004). A number of solutions have been proposed to limit the variability due to leaf surface properties. They include specific measurement configurations such as using an integrating sphere under normal illumination providing directional hemispherical measurements and transmittance measurements (Fourty et al., 1996). Further, specific processing methods are proposed to be applied to the measurements (McClendon \& Fukshansky, 1990; Rondeaux \& Vanderbilt, 1993), exploiting the fact that most surface processes are mainly driven by surface structure and the refractive index of the surface material that varies little with wavelength in the visible and near infrared domains, leading to wavelength independent features (Saunderson, 
1942). However approximations and difficulties in the implementation of these solutions prevent getting accurate and robust estimates of leaf biochemical composition across species, cultivars and environmental conditions.

At the canopy scale, very little attention has been paid to the impact of leaf scattering phase function on canopy reflectance, leaves being generally assumed Lambertian with no differences between faces. However, Chelle (2006) used a simple BRDF (Bi-directional Reflectance Distribution Function) model to evaluate whether the Lambertian assumption was leading to accurate simulations of light absorption in the canopy. He concluded that the effect was marginal, although these results may be highly impacted by the leaf BRDF model used that was not necessarily very realistic. More recently, (Stuckens et al., 2009) demonstrated that the leaf BRDF impacted significantly canopy reflectance over citrus crops with a magnitude that depend on directions and wavelengths, showing that the architecture of the canopy plays also an important role. Nevertheless, the lack of knowledge about leaf anisotropy was already pointed out as one of the main limiting factors in our capacity to accurately describe canopy BRDF (Lewis, 2007).

Bi-directional scattering distribution function (BSDF) allows characterizing the anisotropy of both leaf reflectance (BRDF) and transmittance (BTDF) of the two faces. The early work on leaf BRDF (Howard, 1969) shows a lobe of high reflectance in the forward scattering direction, with a larger magnitude for the larger zenith illumination angles. This is interpreted as resulting from the specular reflectance that was later modeled by Torrance and Sparrow (1967) assuming the leaf surface made of facets with a given distribution of their orientation. The model was later adapted by Bousquet et al. (2005) who included mutual masking created by leaf roughness. However, some leaves showing particular BRDF features such as cereals could not be faithfully modeled using these assumptions (Combes et al., 2007). Other authors have identified a hot-spot in the backscattering direction originating from the mutual shadowing of the surface roughness features (Howard, 1969).

Most experimental studies on leaf BSDF were done using a goniometer where the illumination source and/or the sensor are rotating around the leaf sample (Combes et al., 2007; Walter-Shea et al., 1989). However, these measurements take time with consequences on leaf state mainly because of the desiccation during the experiment (Brakke et al., 1989). Further, difficulties to sample the hot-spot direction because of self-shadowing between the source and the sensor and the increased footprint for the larger view zenith angles limit the angular range. Sarto et al. (1989) proposed an imaging sphere made of a reflective hemisphere that is imaged by a fish-eye camera. This alternative leaf BSDF technique is promising since measurements should be almost instantaneous and with a high angular sampling and resolution. However, this technology developed for specific industrial applications (Rykowski, 2008) has not yet been used for leaf BSDF measurements. Other communities have been very active in developing a new BRDF measurement technique based on Fourier optics. The resulting conoscope system (2004; Ged et al., 2010; Obein et al., 2001), allows unprecedented angular sampling both in terms of range and resolution while measurements are sufficiently rapid to maintain the leaf in good conditions during data acquisition. The objective of this study is to provide a detailed description of leaf BRDF in the visible domain over wheat leaves using the conoscope system. Wheat represents the most cultivated crop and several agriculture applications focusing either on precision farming or high throughput phenotyping exploit canopy reflectance measurements for characterizing structural traits such as leaf area index or leaf attributes including chlorophyll content. The measurements achieved aimed at better understanding the main processes governing wheat leaf BRDF and quantifying their contribution to the directional hemispherical reflectance or albedo.

\section{Materials and methods}

The conoscope EZ-Contrast80M designed by Eldim SA (www. eldim.fr) was used in this experiment. It is based on a Fourier optic lens (Bass, 1995; Saleh \& Teich, 1991) that projects the angular distribution $\left(\theta_{\nu}, \varphi_{\nu}\right)$ of the reflected radiation onto a cooled Charge Coupled Device (CCD) matrix (Fig. 1). A regulated xenon arc light source with a collimated beam (solid angle $<10^{-4} \mathrm{sr}$ ) was passing through a diaphragm with $0.33 \mathrm{~mm}$ diameter aperture, corresponding to the size of the light spot on the leaf. The direction of the incident beam may be manipulated both in zenith and azimuth directions. However, the zenith illumination was fixed at $\theta_{s}=45^{\circ}$ in this study, while the azimuth illumination was set either parallel to the leaf longitudinal direction $\left(\varphi_{s}=0^{\circ}\right)$ or transversally $\left(\varphi_{s}=90^{\circ}\right)$ (Fig. 2). The change between longitudinal to transversal illumination was achieved by rotating the leaf. The size of the illuminated spot on the leaf was slightly larger than the spot sampled by the Fourier optics. Radiance signal is recorded in all azimuth directions and with zenith angles up to $80^{\circ}$ over a $400 \times 400 \mathrm{CCD}$ matrix. The signal is finally transformed into a $80 \times 360$ matrix corresponding to zenith view angles $0^{\circ} \leq \theta_{\nu} \leq 80^{\circ}$ and view azimuth $0^{\circ} \leq \varphi_{\nu} \leq 360^{\circ}$ by $1^{\circ}$ step.

Five spectral filters mounted in front of the sensor matrix (Fig. 1) are combined to compute the XYZ tristimulus values (CIE 1931) used in vision related applications for which the conoscope was originally designed. Each tristimulus coordinate corresponds to the radiance measured in a broad band with a spectral sensitivity shown in Fig. 3. The XYZ coordinates correspond roughly to a Red, Green and Blue bands and we will use this last denomination along this study.

The signal corresponding to each element of the $80 \times 360$ matrix in a given band $\lambda$ is proportional to the luminance of the target, $L\left(\theta_{s}, \varphi_{s}\right.$, $\left.\theta_{v}, \varphi_{v}, \lambda\right)$. The calibration coefficient $\alpha\left(\theta_{s}, \varphi_{s}\right)$ depends only on the illumination geometry, the conoscope being designed to provide repeatable values across the view geometries, i.e. valid for the whole $80 \times 360$ matrix. Further, for a given geometry, the calibration coefficient $\alpha\left(\theta_{s}, \varphi_{s}\right)$ was constant with time during the experiment since the light source and the sensor matrix were stabilized. An experience made using a light trap showed that under the illumination condition $\left(\theta s=45^{\circ}\right)$ the stray light effects were found at the same level than instrumental noise or lower. According to this result, no correction of stray light was applied. A Labsphere Spectralon reference panel was used to transform the measured radiance values into bidirectional reflectance factor, $\operatorname{BRF}\left(\theta_{s}, \varphi_{s}, \theta_{v}, \varphi_{\nu}, \lambda\right)$ the main physical quantity used in this study. Several studies reported that the Spectralon panels were not perfect Lambertian surfaces (Bruegge et al., 2001; Jackson et al., 1992), in agreement with our observations (Fig. 4). The manufacturer of the Spectralon panel provides the directional hemispherical reflectance factor for nadir $\left(\theta_{s}=0^{\circ}\right)$ illumination: $\operatorname{DHRF}_{\text {ref }}\left(0^{\circ}, \lambda\right)=0.991$ for $\lambda$ corresponding to the 3 wavebands considered. Since our illumination configuration is different $\left(\theta_{s}=45^{\circ}\right)$ it was approximated that $D H R F_{r e f}\left(45^{\circ}, \lambda\right) \approx D H R F_{r e f}\left(0^{\circ}, \lambda\right)$, hence assuming that the absorption of the panel is about constant (and very small) up to $\theta_{s}=45^{\circ}$. This was later confirmed by independent measurements made with a goniometer (results not shown for the sake of brevity). In these conditions, the BRF of the leaf is computed as:

$$
\begin{aligned}
& B R F_{\text {leaf }}\left(45^{\circ}, \varphi_{s}, \theta_{\nu}, \varphi_{\nu}, \lambda\right) \\
& =0.991 \frac{L_{\text {leaf }}\left(45^{\circ}, \varphi_{s}, \theta_{\nu}, \varphi_{\nu}, \lambda\right)}{\frac{\sum_{\varphi \nu=0^{\circ}}^{360^{\circ}} \sum_{\theta \nu=0^{\circ}}^{90^{\circ}} L_{\text {ref }}\left(45^{\circ}, \varphi_{s}, \theta_{\nu}, \varphi_{\nu}, \lambda\right) \sin \left(\theta_{\nu}\right) \cos \left(\theta_{\nu}\right)}{\sum_{\theta \nu=0^{\circ}}^{90^{\circ}} \sin \left(\theta_{\nu}\right) \cos \left(\theta_{\nu}\right)}}
\end{aligned}
$$

Where $L_{r e f}\left(45^{\circ}, \varphi_{s}, \theta_{\nu}, \varphi_{\nu}, \lambda\right)$ and $L_{r e f}\left(45^{\circ}, \varphi_{s}, \theta_{\nu}, \varphi_{\nu}, \lambda\right)$ are the signals measured respectively on the leaf and on the reference panel. Because of the absence of measurements above $80^{\circ}$, the integration over the whole range of view zenith angles in Eq. (1) was computed assuming a linear variation with $\theta s$ of the term $L_{r e f}\left(45^{\circ}, \varphi_{s}, \theta_{\nu}, \varphi_{\nu}, \lambda\right)$ $\sin \left(\theta_{\nu}\right) \cos \left(\theta_{\nu}\right)$ for $80^{\circ}<\theta_{s}<90^{\circ}$, taking advantage of the properties 


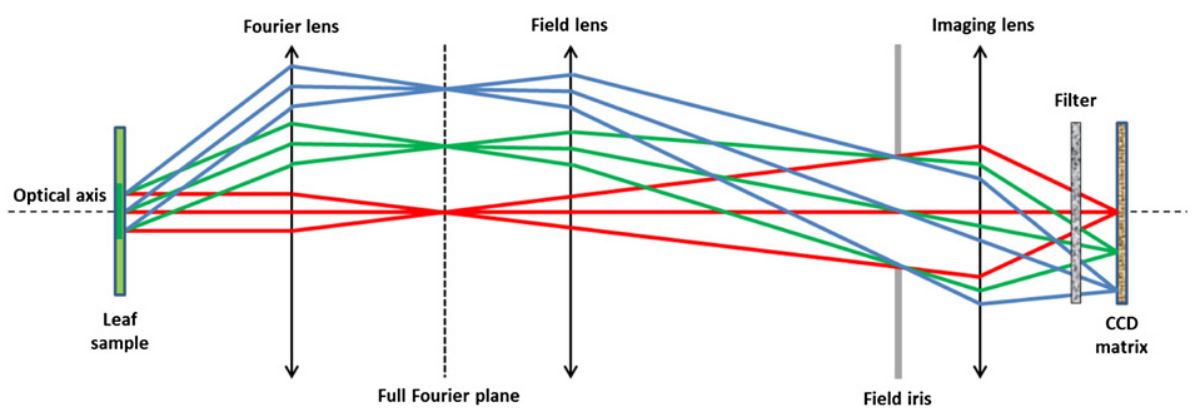

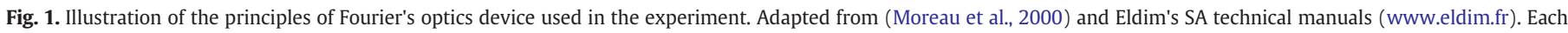

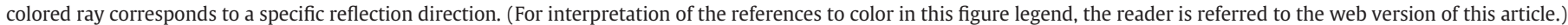

of trigonometric functions: $\theta_{\nu}=90^{\circ} \Rightarrow 90^{\circ} \Rightarrow \cos \left(\theta_{\nu}\right)=0$. The DHRF may be computed from the BRF values (Eq. (2)) using the same approximation for $80^{\circ}<\theta_{s}<90^{\circ}$.

$$
\begin{aligned}
& \operatorname{DHRF}\left(45^{\circ}, \varphi_{s}, \lambda\right) \\
& =\frac{\sum_{\varphi \nu=0^{\circ}}^{360^{\circ}} 90^{\circ}=0^{\circ} B R F\left(45^{\circ}, \varphi_{s}, \theta_{\nu}, \varphi_{v}, \lambda\right) \sin \left(\theta_{v}\right) \cos \left(\theta_{v}\right)}{\sum_{\theta \nu=0^{\circ}}^{90^{\circ}} \sin \left(\theta_{\nu}\right) \cos \left(\theta_{\nu}\right)}
\end{aligned}
$$

Describing the directional variation of BRF is complex because it is a multidimensional problem: $\operatorname{BRF}\left(\theta_{s}, \varphi_{s}, \theta_{\nu}, \varphi_{\nu}, \lambda\right)$ having 5 inputs. When fixing the illumination configuration to $\left(\theta_{s}^{c}, \varphi_{s}^{c}\right)$ and the waveband $(\lambda)$, a 3D polar surface representation (Fig. 4a) provides an intuitive view of $\operatorname{BRF}\left(\theta_{s}^{c}, \varphi_{s}^{c}, \theta_{\nu}, \varphi_{\nu}, \lambda\right)$. In this representation, a perfect Lambertian target should correspond to a hemisphere. Pseudo-3D isocontour lines and gray levels (Fig. 4b) provides a more quantitative view of $\operatorname{BRF}\left(\theta_{s}^{c}, \varphi_{s}^{c}, \theta_{\nu}, \varphi_{\nu}, \lambda\right)$. The Lambertian target should correspond to a uniform gray level disc. Finally, 2D polar representation of cross sections along a given view azimuth $\left(\varphi_{\nu}\right)$ (Fig. 4c) provides both an intuitive view and easy comparison between different view azimuth or other factors such as illumination azimuth $\left(\varphi_{s}\right)$. A Lambertian target should correspond here to a perfect hemi-circle. Note that small variations were observed between the longitudinal and transversal measurements over the spectralon (Fig. 4c), probably due to some particular surface feature of our reference panel. However a very good consistency was observed for the DHRF of the spectralon panel for the 2 azimuthal directions. Consequently, this effect will not impact the accuracy of our calibration procedure since only the DHRF values are used (Eq. (2)).

Measurements were achieved over Triticum durum Isildur wheat cultivar. The plants from which the leaves were sampled were grown in pots in a green-house in good water and nitrogen conditions. Five leaves were collected from the top layers of the plant, selecting the ones that were the most healthy to provide an homogenous sample. On each leaf, the measurements were replicated over three different locations, making an overall sample size of 15 replicates. After the calibration phase that lasted around $10 \mathrm{~min}$, each individual $B R F\left(45^{\circ}, \varphi_{s}, \theta_{\nu}, \varphi_{\nu}, \lambda\right)$ measurement for the three wavebands took about half a minute to be completed. This means that a leaf stayed about $2 \mathrm{~min}$ in the system to complete the measurements at three different locations, minimizing possible changes due to heating or lighting in the system.

\section{Results and discussion}

The variability between the several replicates will first be investigated. Then, detailed description of the BRF directional variation will be presented. A decomposition of the BRF into surface (specular and hot-spot) and volume scattering will be proposed with contribution to the DHRF quantified.

\subsection{Representativeness of measurements}

The BRF directional patterns show a significant variability across the 15 ( 3 points over 5 leaves) replicates for a given illumination direction and the green waveband (Fig. 5). However, similar features may be observed in a given series of 15 measured BRFs. For both longitudinal $\left(\varphi_{s}=0^{\circ}\right)$ and transversal $\left(\varphi_{s}=90^{\circ}\right)$ illumination conditions, a symmetry with regard to the principal plane (the azimuthal plane containing the incident direction) is observed as expected (Fig. 4a). The variability between replicates of BRF measurements is mainly due to the small footprint (about $0.087 \mathrm{~mm}^{2}$ ) as compared to the surface variability mostly created by the longitudinal veins (Fig. 6) with about $0.2 \mathrm{~mm}$ distance between two veins. For a few leaves, the BRF for the larger view zenith angles is very low (Fig. 5a, plot on the left and Fig. 5b, plot on the right). This is possibly due to the fact that the leaf was not always perfectly flat on the sample holder and that some reflected light escaped the Fourier lens for these large angles.

Exploiting the expected symmetry in the BRF with regard to the principal plane for each illumination direction (Fig. 5) allows
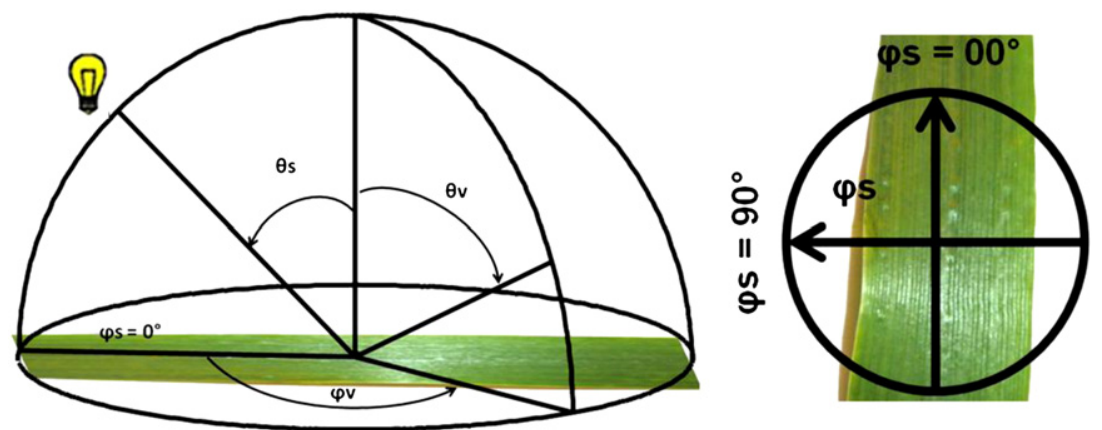

Fig. 2. The geometry of the observation. The azimuth angles $\varphi_{s}$ and $\varphi_{\nu}$ are relative to the orientation of the leaf veins in the longitudinal direction. 


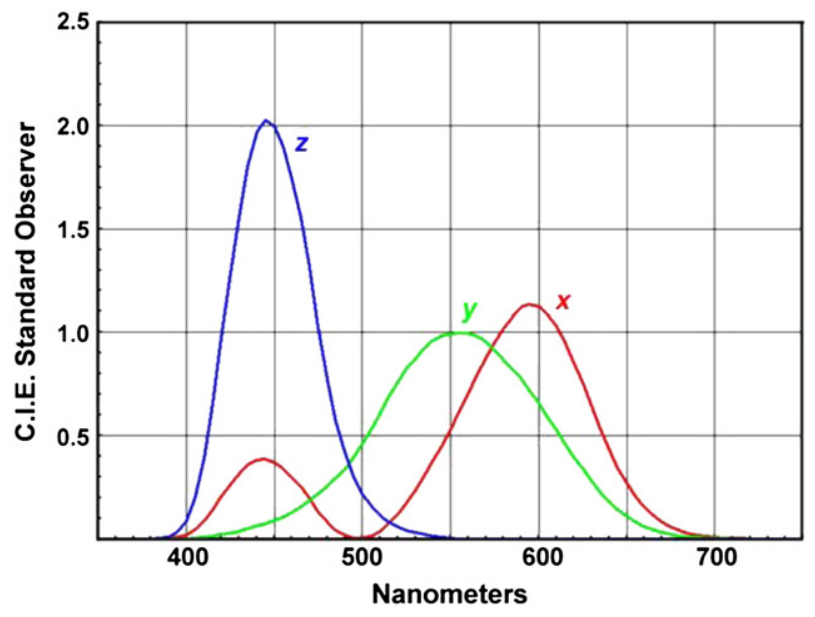

Fig. 3. Spectral sensitivity of the tristimulus coordinates XYZ (from CIE 1931) corresponding roughly to the red, green and blue wavebands. (For interpretation of the references to color in this figure legend, the reader is referred to the web version of this article.)

considering each side as a replicate. This improves the representativeness of the BRF by averaging over 30 samples. The Coefficient of Variation (CV) computed over the 30 replicates (Fig. 7) shows maximum values between $10 \%$ and $75 \%$ for the high view zenith angles $\left(70^{\circ}<\theta_{\nu}<80^{\circ}\right)$. This is due to three factors: (i) the specific design of the conoscope for which fewer photons are captured by the pixels of the CCD matrix for the larger view zenith angles, (ii) the possible shift from the focal plane where the sample should lay affects more the larger view zenith angles, and (iii) the fact that the CV increases mathematically when the BRF decreases as in the case of the larger view zenith angles. Note however that the absolute value of the uncertainty is still small. For view zenith angles $\theta_{\nu} \mathcal{\nu}<70^{\circ}$, the CV increases $(10 \%-50 \%)$ around the principal plane, due to the enhanced sensitivity of the specular scattering on the local roughness features. Outside these directions, the CV is around 10\%-25\%.

The average of all 30 replicates will be used. In the following, corresponding to a total surface sampled of $1.31 \mathrm{~mm}^{2}$.

\subsection{General BRF directional patterns and tentative decomposition}

All BRF patterns show a very pronounced anisotropy, mainly characterized by a large increase of reflectance in the forward scattering direction corresponding to the specular reflection (Fig. 8). Other features are as well observed, in particular the hot-spot corresponding to the backscattering directions. The contribution to the BRF (and corresponding $D H R F$ ) of several scattering processes will be distinguished assuming that they are additive:

$$
\begin{aligned}
& B R F_{\text {tot }}=B R F_{\text {spec }+}+B R F_{\text {spec }-}+B R F_{\text {hot }}+B R F_{\text {iso }} \\
& D H R F_{\text {tot }}=D H R F_{\text {spec }+}+D H R F_{\text {spec }-}+D H R F_{\text {hot }}+D H R F_{\text {iso }}
\end{aligned}
$$

Where subscripts spec + and spec- correspond respectively the specular component in the forward or backward directions, hot corresponds the hot-spot component observed in the backscattering direction, iso is the isotropic component originating from the volume scattering and tot is the total leaf BRF or DHRF. This decomposition of the BRF into additive terms is very usual in semi-empirical approaches (Li \& Strahler, 1985; Roujean et al., 1992). Note however, that this additive decomposition does not presume physical soundness, but provides a simple way to quantify the main features. In the following each of these components will be described with attention paid to the orientation of the leaf with regard to the illumination direction $\left(\varphi_{s}=0^{\circ}\right.$ or $\left.\varphi_{s}=90^{\circ}\right)$. Since very similar features were observed over the three wavebands, results will be presented only for the green waveband.

\subsubsection{Hotspot}

The hotspot feature corresponds to the enhanced back-scattering observed over rough surfaces due to the limited shadow fraction seen by the sensor. Our measurements clearly show such features with a local maximum BRF value in the illumination direction (Fig. 9). This is in good agreement with previous observations (Howard, 1969) demonstrating that the roughness of the leaf creates a hotspot feature.

The hotspot feature was characterized by its extent and its magnitude. Extent was computed as the solid angle, $\Omega_{h o t}$ centered on the

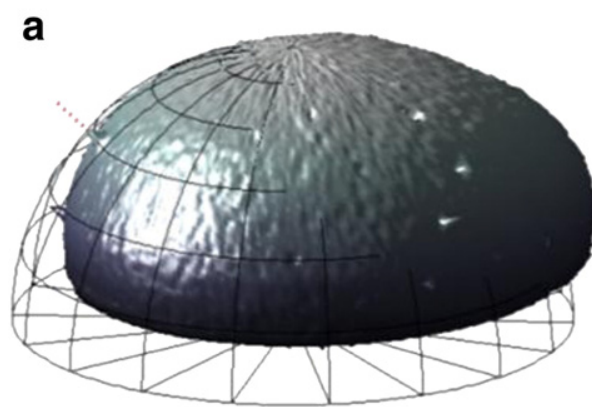

1.25
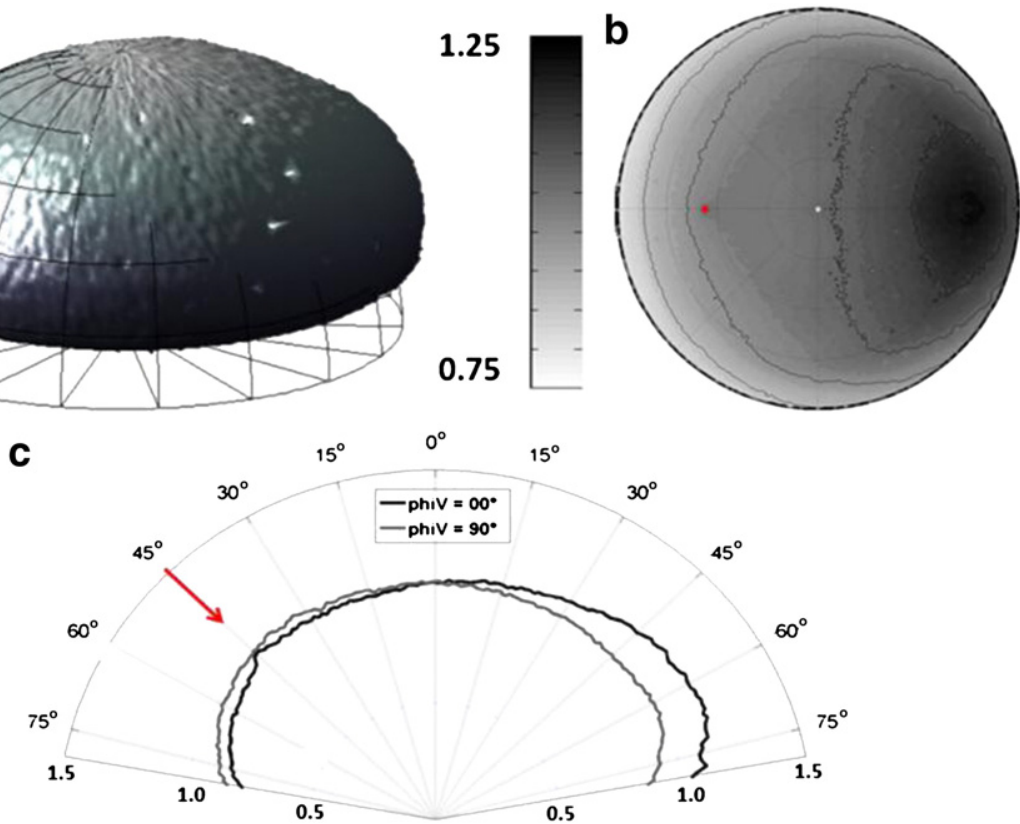

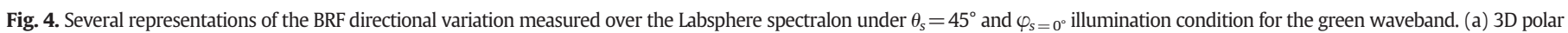

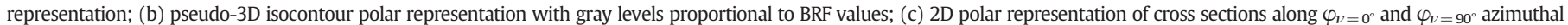

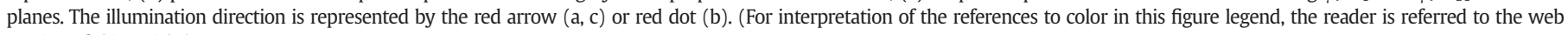
version of this article.) 


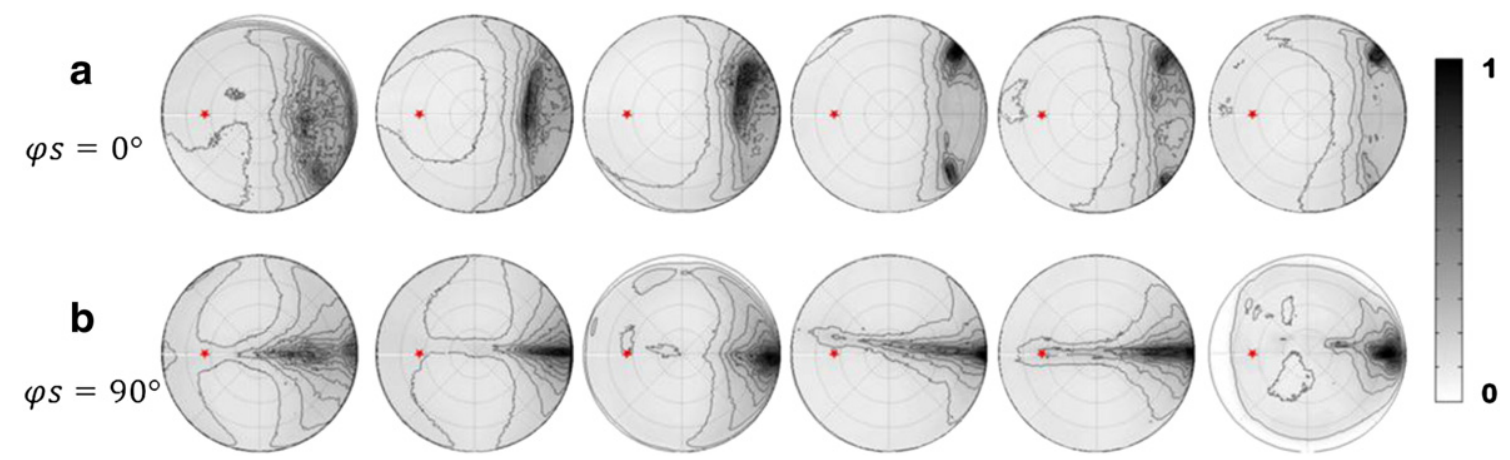

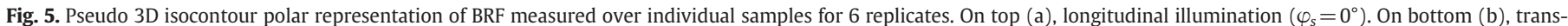
versal illumination $\left(\varphi_{s}=90^{\circ}\right)$. The green waveband is used here.

illumination direction for which the rate of variation of the BRF relative to the angular distance to the illumination direction is larger than $3 \%$ per degree. This threshold was empirically adjusted to retain most of the hot-spot feature while minimizing the contribution of the other surface scattering components characterized by lower local angular rate of variation. Results show that the hotspot angular extent are $\Omega_{\text {hot }}=2.4^{*} 10^{-3} \mathrm{sr}$ and $\Omega_{\text {hot }}=8.6^{*} 10^{-4} \mathrm{sr}$ respectively for longitudinal and transversal illumination directions (Table 1 ). The hotspot magnitude $\left(B R F_{h o t}\left(45^{\circ}, \varphi_{\nu}, 45^{\circ}, \varphi_{\nu}, \lambda\right)\right)$ and contribution of the $\operatorname{DHRF}_{\text {hot }}\left(45^{\circ}, \varphi_{\nu}, \lambda\right)$ to the leaf DHRF $\left(D H R F_{\text {tot }}\left(45^{\circ}, \varphi_{\nu}, \lambda\right)\right)$ were then computed (Table 1) assuming that the sum of the other BRF components varies linearly with the view zenith and azimuth angles within the local hotspot angular extent $\left(\Omega_{\text {hot }}\right)$.

More detailed inspection of the hotspot feature shows differences between the two illumination directions (Table 1). For longitudinal illumination $\left(\varphi=0^{\circ}\right)$, the hotspot is very small in magnitude but with a significant angular extent, in relation to the limited apparent roughness along the direction parallel to the veins. Conversely, in the transversal illumination direction $\left(\varphi_{s}=90^{\circ}\right)$, the hotspot is much sharper, i.e. developed in magnitude but with a limited angular extent, in relation with the larger apparent roughness in the direction across the veins.

Although the hot-spot feature is significant locally in magnitude and extent (Fig. 9), it represents however a negligible contribution to the total hemisphere: the extent represents $0.04 \%$ of the total hemisphere and the contribution of the $\operatorname{DHRF}_{h o t}\left(45^{\circ}, \varphi_{\nu}, \lambda\right)$ is lower than $0.003 \%$ of the $\operatorname{DHRF}_{\text {tot }}\left(45^{\circ}, \varphi_{\nu}, \lambda\right)$ (Table 1$)$.

\subsubsection{Specular reflection}

The specular reflection creates the most prominent BRF feature, with a large increase of the BRF values in the forward scattering direction (Figs. 8 and 10). However, large differences are observed between the two illumination directions. For longitudinal illumination $\left(\varphi_{s}=0^{\circ}\right)$, the specular lobe is widely spread over the azimuth from $-45^{\circ}<\varphi_{\nu}<45^{\circ}$ and for zenith angles $\theta_{\nu}<75^{\circ}$. In this broad specular lobe, the maximum BRF value is about constant and almost independent of $\varphi_{\nu}$ with $\operatorname{BRF}\left(45^{\circ}, 0^{\circ}, \theta_{\nu}^{\operatorname{maxBRF}},-45^{\circ}<\varphi_{\nu}<+45^{\circ}\right) \approx 0.342$ (Fig. 10a). The zenith locations of these maximum BRF values, $\theta_{\nu}^{\operatorname{maxBRF}}$, depend on the azimuth $\varphi_{\nu}$ and is described by $\theta_{\nu}^{\operatorname{maxBRF}}\left(\varphi_{\nu}\right)=2.51-$ $1.785 \cos \left(\varphi_{\nu}\right)$ where angles are expressed in degree, yielding a standard deviation of residuals of 0.002 . This location of maximum BRF values is well described in Fig. 8 by the darkest area as outlined later. For transversal illumination, the specular lobe is more concentrated around the single maximum value $\operatorname{BRF}\left(45^{\circ}, 90^{\circ}, 74^{\circ}\right.$ and $\left.180^{\circ}\right)=0.72$ (Fig. 10b). This specular lobe is still significant for nadir viewing but vanishes in the backward scattering direction (Fig. 10b). The BRF shows a significant 'retro specular' lobe for longitudinal illumination with values higher than 0.13 for view zenith angles ranging from $20^{\circ}$ to $55^{\circ}$. Such 'retro-specular' lobe, distinct from the hot-spot feature, was already observed over fescue grass leaves having longitudinal veins creating an anisotropic roughness (Combes et al., 2007) as for the wheat investigated here. It is generated by the contribution of the facets having their normal pointing in the backward direction.

For longitudinal illumination, light may be scattered primarily by the flanks of the veins, explaining the large specular lobe. Conversely, for transversal illumination, light is primarily scattered close to the principal plane in directions depending on the local inclination of the surface.

\subsubsection{Isotropic component}

Because of the assumed additive nature of the several components (Eq. (3)), the isotropic component could be approximated by the

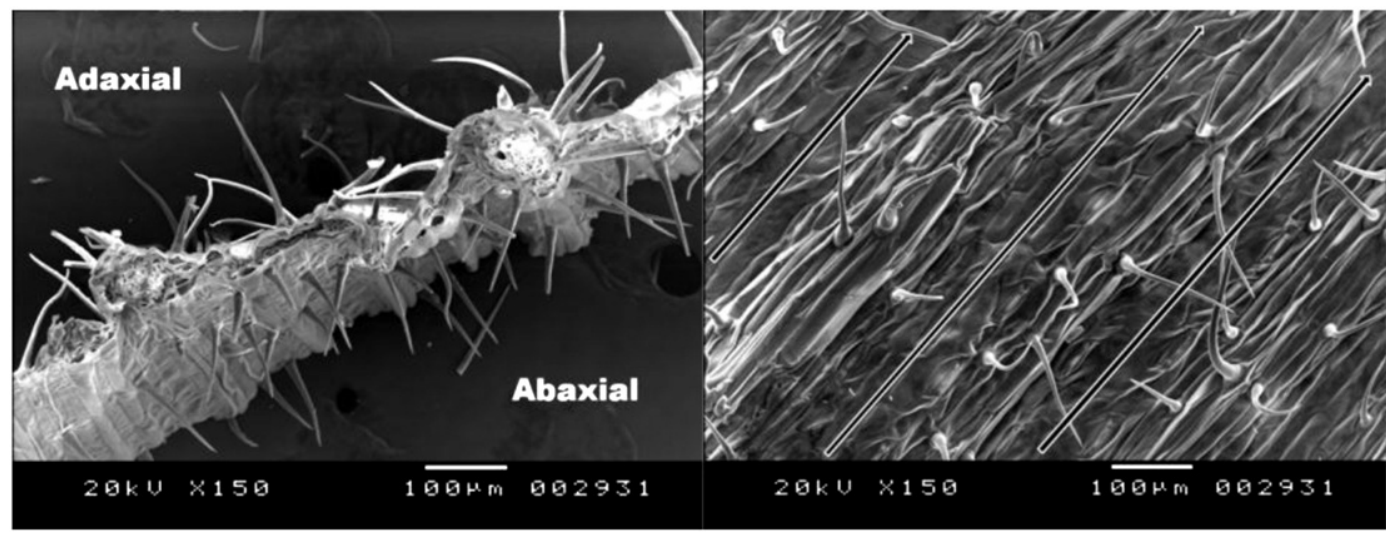

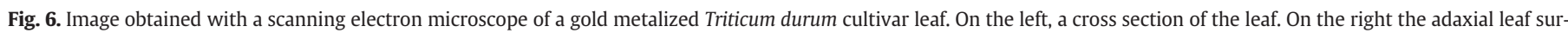
face as seen from nadir. Black and white arrows are in-between the veins and oriented along the longitudinal axis of the leaf. 

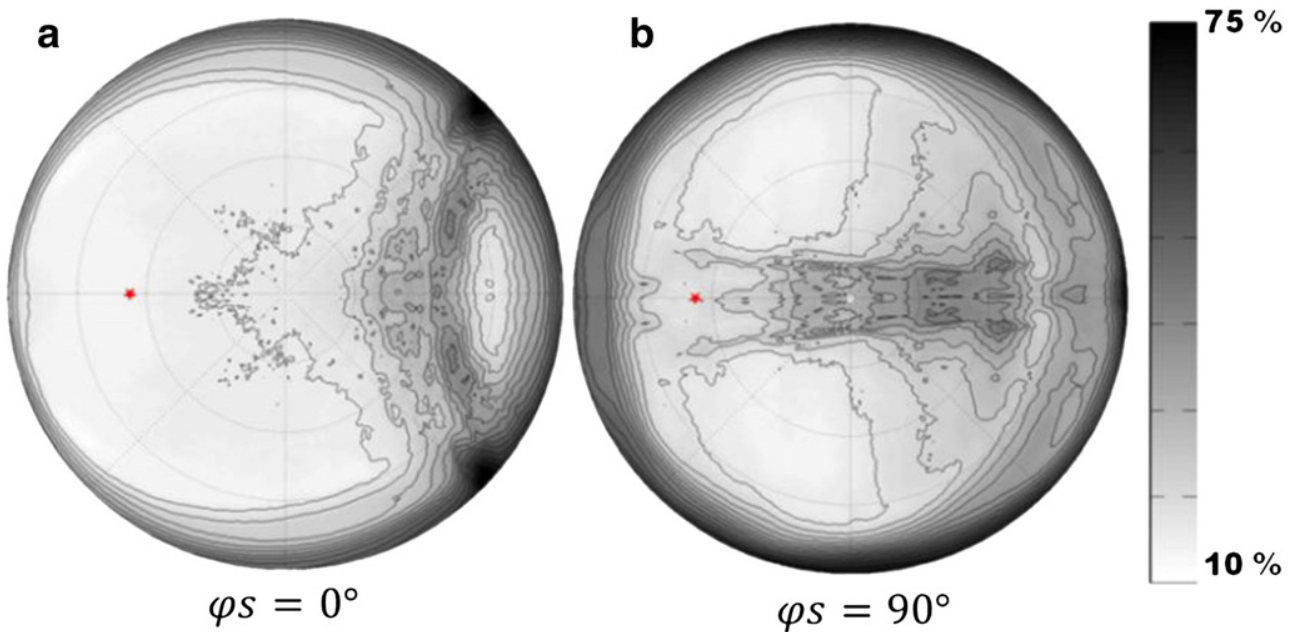

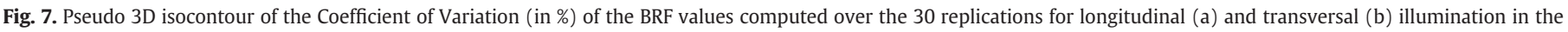
green band.

minimum BRF value where the other scattering components are minimal: $\operatorname{DHRF}_{i s o}\left(45^{\circ}, \varphi_{s}\right) \approx \min \left(\operatorname{BRF}\left(45^{\circ}, \varphi_{s}, \theta_{\nu}, \varphi_{\nu}\right)\right)$.

Rather than taking the minimum measured BRF value that could correspond to some noise, the isotropic component value was set to that corresponding to a low frequency threshold value in the BRF cumulated distribution (Fig. 11). The relatively flat distribution observed for the lower BRF values before the steep increase of the cumulated frequency (Fig. 11) indicates that the estimated isotropic value will be little sensitive to the threshold frequency $f$. As a matter of fact, $(\operatorname{BRF}(f=10 \%)-\operatorname{BRF}(f=4 \%))<0.004$. The threshold was therefore set to $f=5 \%$, leading to $\mathrm{BRF}_{\text {iso }}\left(45^{\circ}, 0^{\circ}\right)=0.082$ and $\mathrm{BRF}_{\text {iso }}\left(45^{\circ}, 90^{\circ}\right)=$ 0.095 for the isotropic component values.

The lowest BRF values are observed for the larger view zenith angles for transversal illumination (Fig. 12). However, these large view zenith angles are associated to higher noise levels and possible artifacts as discussed earlier. The directions $\left[\theta_{\nu}, \varphi_{\nu}\right]$ where the minimum BRF values are observed and with $\theta_{\nu}>70^{\circ}$ depend on the illumination conditions: for longitudinal illumination, the minimum BRF values are located at $10^{\circ}<\theta_{\nu}<30^{\circ}$ with $-45^{\circ}<\varphi_{\nu}<45^{\circ}$ (Fig. 12a) with $B R F_{\text {iso }}\left(45^{\circ}, 0^{\circ},-,-\right)=D H R F_{\text {iso }}\left(45^{\circ}, 0^{\circ}\right) \approx 0.082$; for transversal illumination, the minimum values are located at $30^{\circ}<\theta_{\nu}<50^{\circ}$ with $\left(-40^{\circ}<\varphi_{\nu}<10^{\circ}\right)$ (Fig. 12b) with $\operatorname{BRF}_{\text {iso }}\left(45^{\circ}, 90^{\circ},-,-\right)=D H R F_{\text {iso }}\left(45^{\circ}\right.$, $\left.90^{\circ}\right) \approx 0.095$.

\subsection{Spectral variation of BRF and DHRF}

After focusing on the BRF features of the green waveband, the spectral dependency of the scattering components of the DHRF will be investigated. However, since the hotspot contribution to the DHRF was demonstrated to be very small, it will be neglected here
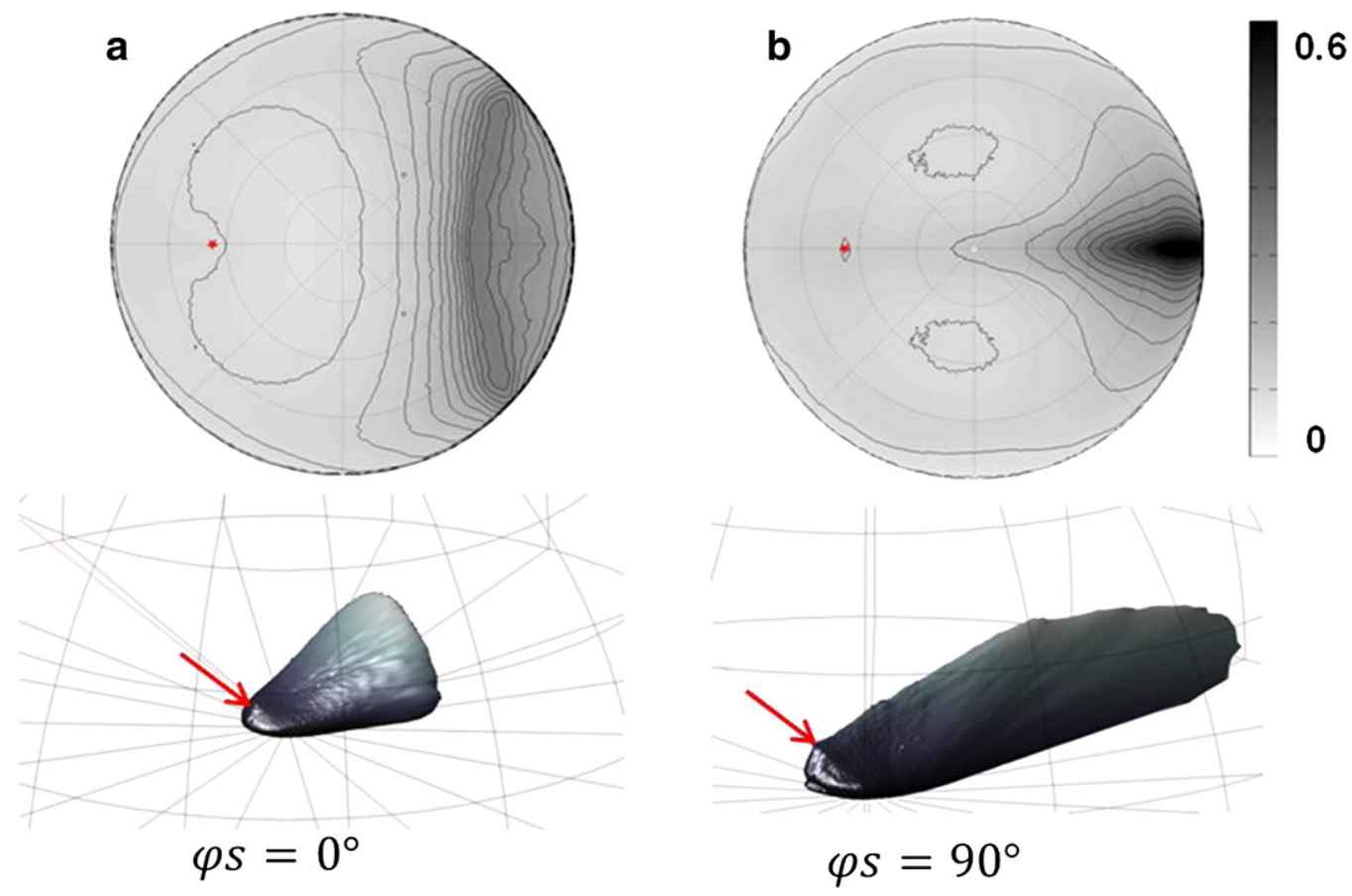

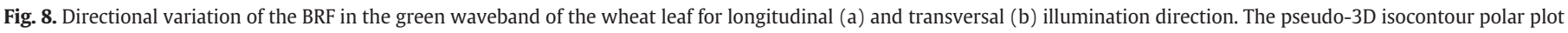

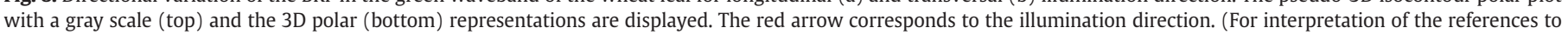
color in this figure legend, the reader is referred to the web version of this article.) 


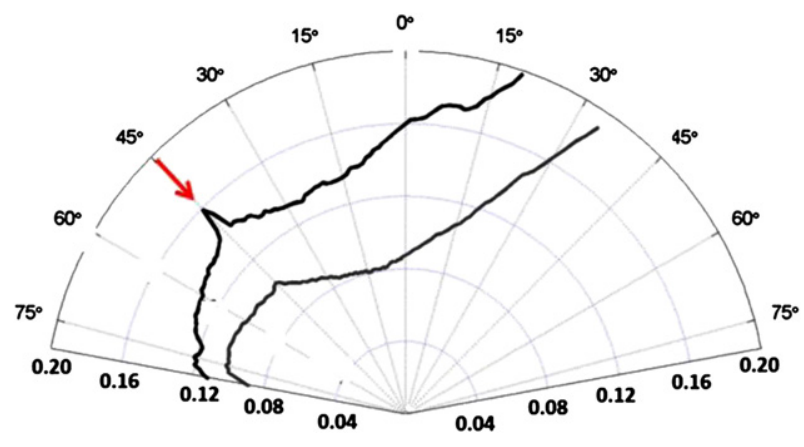

Fig. 9. BRF directional variation with $2 \mathrm{D}$ polar representation in the principal plane $\left(\varphi_{\nu}=\left[0^{\circ}, 180^{\circ}\right]\right)$ with emphasis on the illumination direction (red arrow) for longitudinal $\left(\varphi_{s}=0^{\circ}\right)$ in gray and transversal $\left(\varphi_{s}=90^{\circ}\right)$ in black illumination. The green waveband is used here. (For interpretation of the references to color in this figure legend, the reader is referred to the web version of this article.)

and emphasis will be put on of the specular and isotropic components. Finally, the isotropic component will be tentatively related to the volume scattering.

\subsubsection{Quantification of DHRF components and their spectral dependency}

The several DHRF components were computed from the BRF measurements using Eq. (3) and the approximation of the isotropic component as defined by the minimum BRF values. Results show that the surface scattering $\left(D H R F_{\text {surf }}=D H R F_{\text {spec }+}+D H R F_{\text {spec- }}\right)$ does not depend on wavelength, with $\operatorname{DHRF}_{\text {surf }}\left(45^{\circ}, 0^{\circ}\right)=0.048$ and $D H R F_{\text {surf }}$ $\left(45^{\circ}, 90^{\circ}\right)=0.037$ respectively for longitudinal and transversal illuminations, representing respectively $42 \%$ and $31 \%$ of the leaf $\mathrm{DHRF}_{\text {tot }}$ (Table 2 and Fig. 13). This expected result is in good agreement with those of Bousquet et al. (2005). The independency of the specular component with wavelength comes from the small change of the spectral refraction index that drives specular reflection in the visible wavelength. The forward-specular component is dominant with $92 \%$ (longitudinal) and $84 \%$ (transversal) of the total surface component. The retro-specular component represents thus only a small fraction of the total specular scattering with $8 \%$ (longitudinal) and $16 \%$ (transversal) of the surface scattering component. The oriented roughness of the leaf surface explains these differences: more facets are illuminated under grazing angles in the longitudinal illumination, generating a very broad specular component. Conversely, for transversal illumination, a lower fraction of facets are illuminated under such conditions, mainly concentrated on the top of the veins and creating a strong but sharp specular lobe as described previously. Further, for transversal illumination, a significant number of facets oriented 'backwards' induces the larger retro-specular component than in the longitudinal illumination case.

The isotropic component varies both with wavelength and with the illumination direction (Table 2 and Fig. 13). It shows a maximum in the green while blue and red components are significantly lower. This spectral pattern is easily explained by the fact that the isotropic component originates from the volume scattering mainly driven by chlorophyll absorption. A smaller isotropic component is observed

Table 1

Characteristics of the hot-spot feature for longitudinal $\left(\varphi_{s}=0^{\circ}\right)$ and transversal illumination $\left(\varphi_{s}=90^{\circ}\right)$ in the green waveband.

\begin{tabular}{lll}
\hline & $\varphi_{s}=0^{\circ}$ & $\varphi_{s}=90^{\circ}$ \\
\hline Magnitude: $B R F_{\text {hot }}\left(45^{\circ}, 0^{\circ}, 45^{\circ}, \varphi_{\nu}, \lambda\right)$ & 0.0046 & 0.0113 \\
Extent: $\Omega_{\text {hot }}$ in steradians & 0.0024 & 0.00086 \\
$D H R F_{\text {hot }}$ & $1.7 \times 10^{-6}$ & $3.4 \times 10^{-6}$ \\
$D H R F_{\text {hot }} / D H R F_{\text {tot }}(\%)$ & 0.0013 & 0.0026 \\
\hline
\end{tabular}
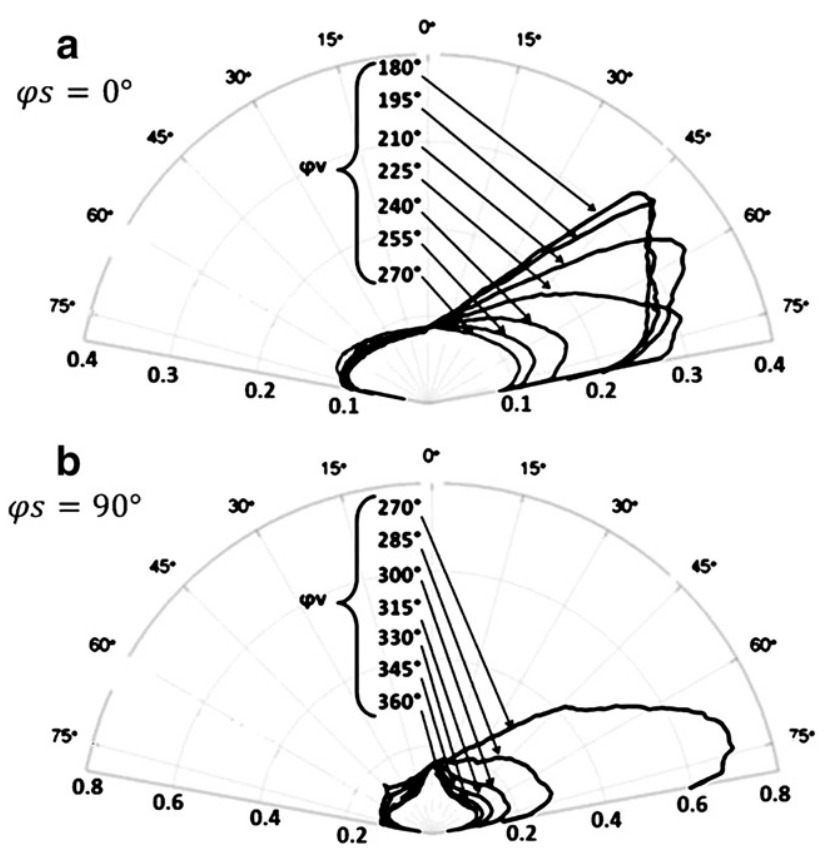

Fig. 10. BRF directional variation with $2 \mathrm{D}$ polar representation in a range of view azimuthal $\left(\varphi_{\nu}\right)$ planes and for the green waveband and for longitudinal (a) and transversal illumination directions. Note that the BRF scale was adapted for each illumination direction.

for longitudinal illumination as compared to the transversal case, compensating almost exactly the difference in surface scattering and resulting eventually in equal hemispherical reflectance: $D H R F_{\text {tot }}$ $\left(45^{\circ}, 0^{\circ}, \lambda\right) \approx D H R F_{\text {tot }}\left(45^{\circ}, 0^{\circ}, \lambda\right)$. The enhanced isotropic component experienced under transversal illumination may be explained by the more light available for volume scattering because of the higher transmittance of the surface due to the lower directional hemispherical reflectance: $\left(D H R F_{\text {surf }}\left(45^{\circ}, 0^{\circ},-\right)-D H R F_{\text {surf }}\left(45^{\circ}, 90^{\circ},-\right)\right) / D H R F_{\text {surf }}$ $\left(45^{\circ}, 0^{\circ},-\right)=30 \%$. The mechanism that links the isotropic component to the volume scattering will be further explained in the following section.

\subsubsection{From the isotropic to the volume scattering}

The isotropic component is assumed to originate from the scattering within the leaf volume. As a matter of fact, multiple scattering within the leaf will provide more randomness in the direction of the scattered radiation resulting in more isotropic reflected and transmitted fluxes. This was confirmed by the several studies reporting relatively isotropic transmittance of leaves (Breece \& Holmes, 1971; Greinier et al., 2007; Sanz et al., 1997). This is also partly justified by the relatively isotropic leaf reflectance in the near infrared domain (Greinier et al., 2007) where volume scattering is dominant because of the very small absorption experienced within the leaf. Under these assumptions, the total leaf DHRF can be computed considering hemispheric fluxes interacting with the leaf surface as sketched in Fig. 14:

$$
\begin{aligned}
& \operatorname{DHRF}_{\text {tot }}\left(45^{\circ}, \varphi_{s}, \lambda\right)=\operatorname{DHRF}_{\text {surf }}\left(45^{\circ}, \varphi_{s}\right)+ \\
& \left(1-\operatorname{DHRF}_{\text {surf }}\left(45^{\circ}, \varphi_{s}\right)\right) B H R F_{\text {vol }}(\lambda) \frac{\left(1-B H R F_{\text {surf }}^{\text {in }}\right)}{\left(1-B H R F_{\text {surf }}^{\text {in }} \cdot B H R F_{\text {vol }}(\lambda)\right)} .
\end{aligned}
$$

Where $D H R F_{\text {surf }}\left(45^{\circ}, \varphi_{s}\right)$ corresponds to the surface component originating mainly from the specular reflection, $B H R F_{v o l}(\lambda)$ is the bihemispherical reflectance factor originating from volume scattering, $B H R F_{\text {surf }}^{\text {in }}$ corresponds to the bi-hemispherical reflectance factor due 


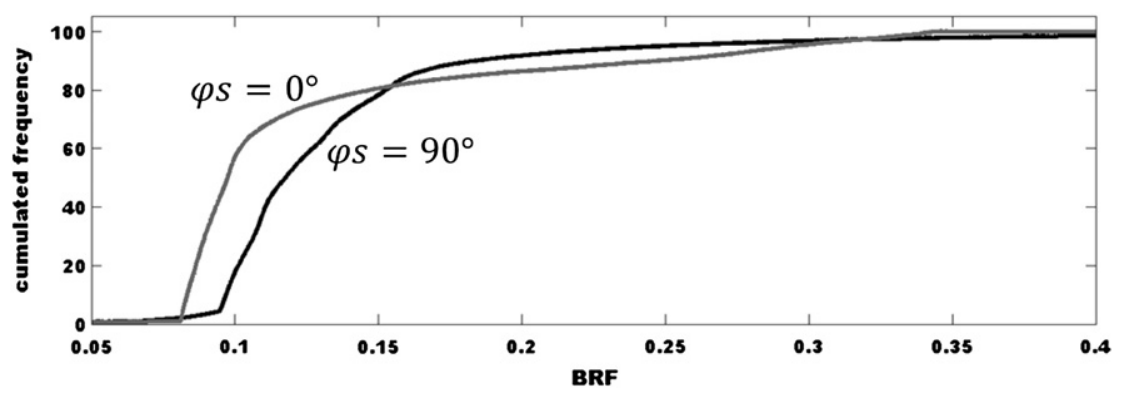

Fig. 11. Cumulated frequency of BRF values computed for longitudinal $\left(\varphi_{s}=0^{\circ}\right.$, in gray) and transversal $\left(\varphi_{s}=90^{\circ}\right.$ in black) illumination directions and the green waveband.
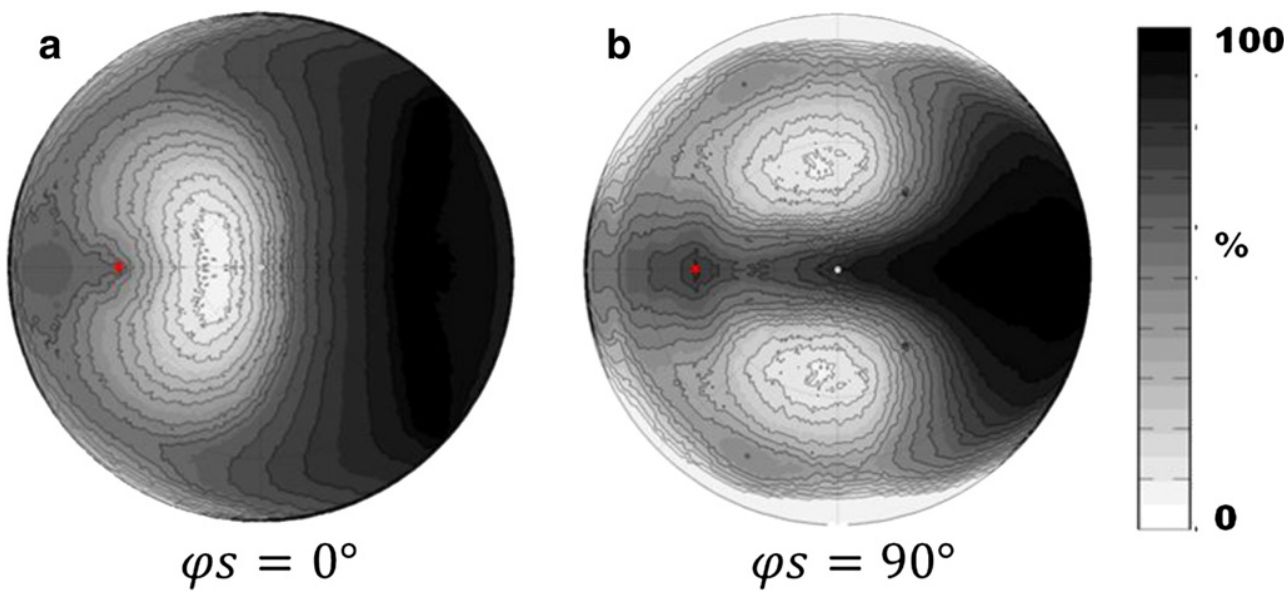

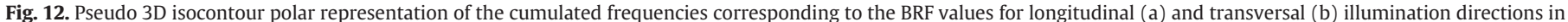
the green waveband.

to scattering at the interface between the leaf volume and the leaf inner surface. It is assumed wavelength independent because it depends mainly on the refraction index and on the surface roughness. Further, the same value of $B H R F_{\text {surf }}^{\text {in }}$ is assumed for the two illumination conditions since isotropic fluxes are considered after the first interaction of the incident light with the surface. The term $\left(1-D H R F_{\text {surf }}\right.$ $\left.\left(\theta_{s}, \varphi_{s}\right)\right)$ in Eq. (4), corresponds to the hemispherical transmittance of the surface for the directional incident radiation, $\left(1-\mathrm{BHRF}_{\text {surf }}^{\text {in }}\right)$ to the hemispherical transmittance for the upward hemispherical flux originating from the volume scattering, and the term $\frac{1}{1-B H R F_{\text {surf }} f^{n} \cdot B H R F_{\text {vol }}(\lambda)}$ accounts for multiple scattering between the leaf volume and the surface. Note that this term is actually very close to 0.999 in our case, i.e. multiple scattering is negligible between the surface layer and the leaf volume (Fig. 14).

Values of $B H R F_{\text {surf }}^{\text {in }}$ and $B H R F_{\text {vol }}(\lambda)$ were estimated by minimizing a cost function using the simplex algorithm (Nelder \& Mead, 1965). The cost function, $J$, is computed over the two illumination conditions and the three wavebands as the quadratic sum of the differences between the measured $D H R F_{\text {tot }}\left(45^{\circ}, \varphi_{s}, \lambda\right)$ and the estimated value $D H R F_{\text {tot }}$ $\left(45^{\circ}, \varphi_{s}, \lambda\right)$ using Eq. (4):

$J=\sum \begin{aligned} & \varphi s=\left[0^{\circ}, 90^{\circ}\right] \\ & =[\text { Blue }, \text { Green, Red }]\end{aligned}\left(D H R F_{\text {tot }}\left(45^{\circ}, \varphi_{s}, \lambda\right)-D H R F_{\text {tot }}\left(45^{\circ}, \varphi_{s}, \lambda\right)\right)^{2}$

Where $\lambda=[$ Blue, Green, Red] corresponds to the three wavebands considered. Results show that the estimated values of $D H R F_{\text {tot }}$ $\left(45^{\circ}, \varphi_{s}, \lambda\right)$ are very close to the actual ones, with RMSE $=0.006$ (Fig. 15). However, a slight overestimation (underestimation) is observed for longitudinal (respectively transversal) illumination. This may result from the several assumptions used, with probably an important contribution to the assumed isotropy of the volume scattering and the approximation of $D H R F_{i s o}$ as the minimum value of the measured BRDF. The retrieved volume component, $B H R F_{\text {vol }}$, are respectively $0.065,0.095$ and 0.075 for the blue, green and red wavebands. The retrieved value of the reflectivity

Table 2

DHRF scattering components. The backward specular one $\left(D H R F_{s p e c-}\right)$ includes the hotspot which is however negligible.

\begin{tabular}{|c|c|c|c|c|c|c|c|c|}
\hline & \multicolumn{4}{|c|}{$\varphi_{s}=0^{\circ}$} & \multicolumn{4}{|c|}{$\varphi_{s}=90^{\circ}$} \\
\hline & Red & Green & Blue & Average & Red & Green & Blue & Average \\
\hline DHRF $F_{\text {spec }+}$ & 0.042 & 0.045 & 0.045 & $0.044(38.2 \%)$ & 0.030 & 0.031 & 0.031 & $0.031(26.0 \%)$ \\
\hline$D H R F_{\text {spec- }}$ & 0.004 & 0.004 & 0.005 & $0.004(3.8 \%)$ & 0.006 & 0.006 & 0.006 & $0.006(5.1 \%)$ \\
\hline$D_{H R F_{\text {surf }}}$ & 0.046 & 0.048 & 0.050 & $0.048(41.6 \%)$ & 0.036 & 0.037 & 0.038 & $0.037(31.4 \%)$ \\
\hline$D_{\text {DRF }}$ iso & 0.064 & 0.082 & 0.055 & $0.067(58.1 \%)$ & 0.078 & 0.097 & 0.068 & $0.081(68.6 \%)$ \\
\hline$D_{H R F_{\text {tot }}}$ & 0.110 & 0.131 & 0.105 & $0.115(100 \%)$ & 0.114 & 0.134 & 0.106 & $0.118(100 \%)$ \\
\hline
\end{tabular}




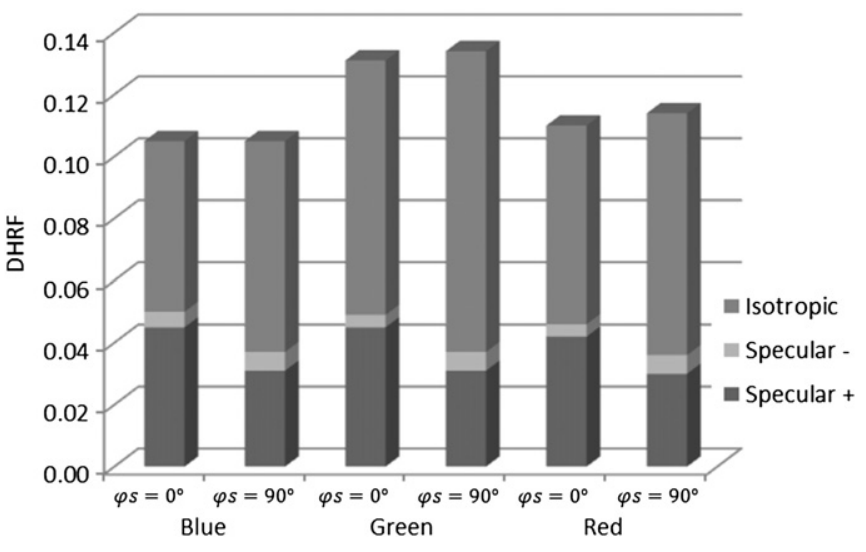

Fig. 13. The DHRF components (Eq. (3)) as a function of the wavebands and illumination directions. The hotspot contribution is here neglected. The data correspond to Table 2.

of the inner surface is $B H R F_{\text {surf }}^{\text {in }}=0.013$, which is about 3 to 4 times lower than the $\operatorname{DHRF}_{\text {surf }}\left(45^{\circ}, \varphi_{s}\right)$.

However, the simple model corresponding to Eq. (4) does not allow to explain the fact that almost the same total leaf $D H R F_{\text {tot }}$ was observed for the two illumination conditions. A first order approximation (i.e. neglecting multiple scattering term between the surface layer and the leaf volume) of the difference $\triangle D H R F_{\text {tot }}$ created by a difference

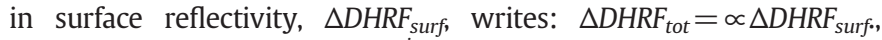
with $\propto \approx\left(1-B H R F_{\text {vol }}\right)\left(1-B H R F_{\text {surf }}^{\text {in }}\right)$. This factor varies between $0.92<\propto<0.89$ when using the $B H R F_{\text {vol }}$ and $B H R F_{\text {surf }}^{\text {in }}$ values estimated for the wavebands considered. This shows that the simple model proposed does not allow to explain the almost insensitivity of $D H R F_{\text {tot }}$ to $D H R F_{\text {sirf }}$, i.e. $\propto \approx 0$. It is probable that the assumption on the independency of $B H R F_{\text {vol }}$ to $B H R F_{\text {surf }}$ is not realistic along with the other assumptions already discussed above.

\section{Conclusions and perspectives}

This paper presents a unique set of BRF measurements over wheat leaves with an unprecedented angular resolution and sampling for the direction of observations. The data are available on EMMAH laboratory website at the following webpage: http://www4.paca.inra.fr/ emmah_eng/Production-Documentation/Database-Satellite-products/ Databases/LeafBRDF. Results obtained under a $45^{\circ}$ zenith incidence angle demonstrated that wheat leaf reflectance is highly anisotropic, with a strong forward scattering corresponding to a specular lobe. The leaf surface roughness created by the longitudinal parallel veins explained very well the variation of the directional scattering features including a small but significant hotspot. The longitudinal and transversal illumination directions experienced show very contrasted BRF

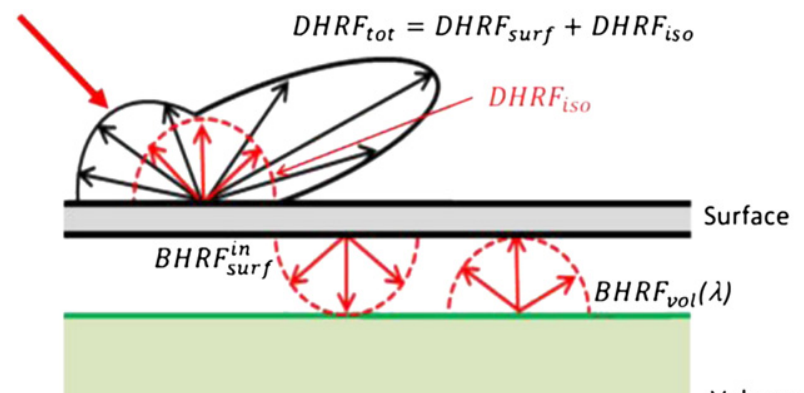

Volume

Fig. 14. Schematic representation of the model used to compute the leaf total DHRF $\left(D H R F_{\text {tot }}\right)$ from the surface scattering $B H R F_{\text {surf }}$, the volume scattering $B H R F_{\text {vol }}$ and the epidermis BHRF (BHRF surf). patterns. In these conditions, models assuming the surface made of elementary facets with normal orientation characterized by a given distribution law for the zenith angles and uniformly distributed azimuth such as proposed by Bousquet et al. (2005), will not be able to simulate realistically the wheat leaf $B R F$.

The whole BRF was integrated over the view directions to compute the DHRF that was tentatively split into a specular, a hotspot and an isotropic component. While the contribution of the hotspot to the total DHRF was negligible, the specular feature was representing about 30\% in the forward scattering and around 5\% in the backward scattering. The specular contribution was found to be mainly independent from the wavelength as expected. The isotropic component was therefore contributing the more to leaf scattering with about $65 \%$ of the leaf DHRF. This isotropic component was wavelength dependent as expected since it originates from the scattering within the leaf volume where absorption occurs. The DHRF was almost independent on the illumination azimuth conversely to the important differences observed on the BRF between longitudinal and transversal illumination. This property might be exploited to access leaf biochemistry from reflectance measurements by minimizing leaf orientation effects within a leaf clip system. It might be achieved either by using an integrating sphere or by deriving the DHRF from a sample of BRF measurements in few well selected directions.

A simple model was proposed to estimate the volume scattering from the isotropic component, taking into account the transmittance of the surface. However, the assumptions made need to be verified, including those relative to the isotropy of the volume scattering and the approximation of a negligible surface contribution where the total BRF is minimum. This model does not explain the fact that the total DHRF of the leaf was almost the same for the two illumination directions despite the differences observed in the surface DHRF between the two illumination directions. A more physically based model that explicitly accounts for the surface roughness of the leaf, its anisotropy and its coupling with the leaf volume needs therefore to be developed. Such model would allow better quantifying the factors that drive the variability of the BSDF between cultivars and environmental conditions, with possible application on the identification of stresses as well as characterization of functional traits for plant phenotyping. Additionally, differences between species are likely to be very important. The proposed physical model should therefore

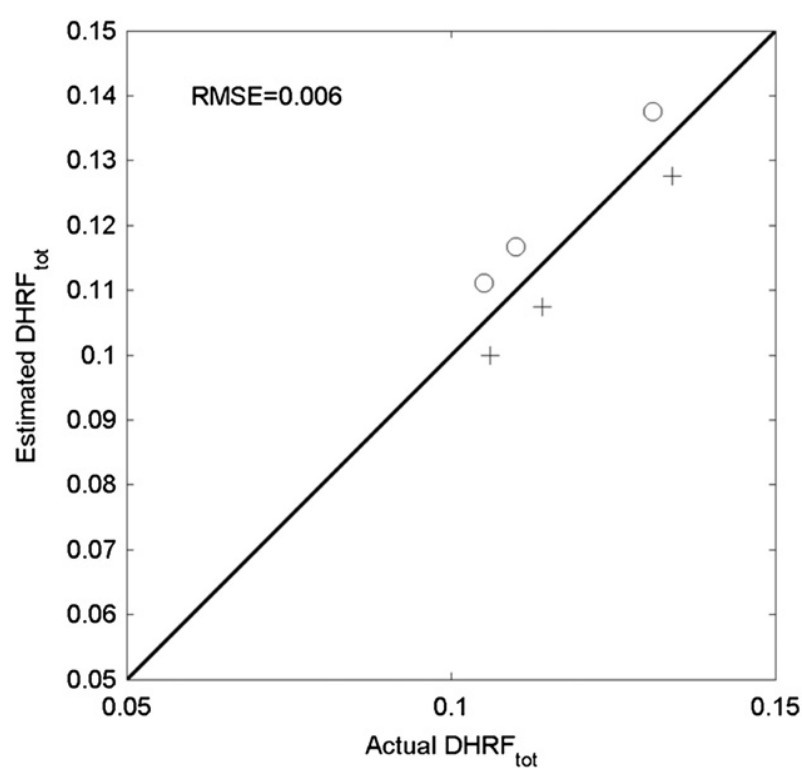

Fig. 15. Comparison between the actual $D H R F_{\text {tot }}\left(45^{\circ}, \varphi_{s}, \lambda\right)$ as computed from measurements according to Eq. (3), and the estimated values with the adjusted $B H R F_{\text {surf }}^{\text {in }}$ and $B H R F_{\text {vol }}$ values. The solid line corresponds to the $1: 1$ line, the 'o' to longitudinal illumination and '+' to transversal illumination. 
have the capability to describe the BSDF over very different leaf types, with variability in surface aspect as well as mesophyll structure and distribution of absorbers such as chlorophyll.

The measurements made in this study were achieved on a single cultivar of wheat, in few visible broad bands and under a unique incident zenithal direction. They are therefore too limited to calibrate or validate a physically based BSDF model. Although there are no technical problems to extend these measurements to few other bands, especially in the near infrared where the mesophyll structure is expected to play an important role (Slaton et al., 2001) as well as to other incidence directions, this requires significant efforts that need to be pursued. Further, transmittance measurements would be also very useful since it would probably reveal some anisotropy as already observed by (Brakke et al., 1989; Breece \& Holmes, 1971; Greinier et al., 2007; Sanz et al., 1997). The understanding of the surface contribution is a very important issue that would allow accessing more accurately the volume scattering that provides the information requested for estimating the biochemical leaf composition.

The knowledge of the leaf BSDF is potentially important for the modeling of canopy light regime. However, the consequences of leaf anisotropy on canopy level radiative quantities are not clear because of the variability of leaf orientation within a canopy, both in zenith and azimuth. Detailed simulations of canopy BRDF made with radiative transfer models with several assumptions on leaf BSDF and canopy structure (leaf area index, leaf orientation ...) should be completed.

\section{Acknowledgments}

This study was part of the 'SURFEUILLE' project supported by the French National Remote Sensing Programme. The first author was supported by a CIFRE grant cofounded by Arvalis Institut du vegetal and the Agence Nationale pour la Recherche (ANR). Many thanks to Gwenaelle Barilliet who have grown the wheat plants as well as to Oulfa Belhadj-Khlaifi who helped us acquire reliable MEB pictures.

\section{References}

Bass, M. (1995). The Optical Society of America: Handbook of Optics. (2nd ed). Chapter 17: Microscope: Orthoscopic vs. Conoscopic Imaging Vol 2, New York: McGraw-Hill.

Bousquet, L., Lacherade, S., Jacquemoud, S., \& Moya, I. (2005). Leaf BRDF measurements and model for specular and diffuse components differentiation. Remote Sensing of Environment, 98, 201.

Brakke, T. W., Smith, J. A., \& Harnden, J. M. (1989). Bidirectional scattering of light from tree leaves. Remote Sensing of Environment, 29, 175-183.

Breece, H. T., \& Holmes, R. A. (1971). Bidirectional scattering characteristics of healthy green soybeans and corn leaves in vivo. Applied Optics, 10,119-127.

Bruegge, C., Chrien, N., \& Haner, D. (2001). A spectralon BRF data base for MISR calibration applications. Remote Sensing of Environment, 76, 354-366.

Chelle, M. (2006). Could plant leaves be treated as Lambertian surfaces in dense crop canopies to estimate light absorption? Ecological Modelling, 198, 219-228.

Combes, D., Bousquet, L., Jacquemoud, S., Sinoquet, H., Varlet-Grancher, C., \& Moya, I. (2007). A new spectrogoniophotometer to measure leaf spectral and directional optical properties. Remote Sensing of Enviroment, 109, 107-117.

Fourty, T., \& Baret, F. (1997). Vegetation water and dry matter contents estimated from top of the atmosphere reflectance data: a simulation study. Remote Sensing of Environment, 61, 34-45.

Fourty, T., Baret, F., Jacquemoud, S., Schmuck, G., \& Verdebout, J. (1996). Leaf optical properties with explicit description of its biochemical composition: direct and inverse problems. Remote Sensing of Environment, 56, 104-117.

Ged, G., Obein, G., Silvestri, Z., Le Rohellec, J., \& Viénot, F. (2010). Recognizing real materials from their glossy appearance. Journal of Vision, 10.

Gitelson, A. A., Gritz, Y., \& Merzlyak, M. N. (2003). Relationships between leaf chlorophyll content and spectral reflectance and algorithms for non-destructive chlorophyll assessment in higher plant leaves. Journal of Plant Physiology, 160, 271-282.
Grant, L., Daughtry, C. S. T., \& Vanderbilt, V. C. (1987). Variations in the polarized leaf reflectance of sorghum bicolor. Remote Sensing of Environment, 21, 333-339.

Greinier, M. A., Duncan, B. D., \& Dierking, M. P. (2007). Bidirectional scattering distribution functions of maple and cottonwood leaves. Applied Optics, 46, 6485-6494.

Holmes, M. G., \& Keiller, D. R. (2002). Effects of pubescence and waxes on the reflectance of leaves in the ultraviolet and photosynthetic wavebands: a comparison of a range of species. Plant, Cell \& Environment, 25, 85-93.

Howard, J. A. (1969). Increased luminance in the direction of reflex reflection - a recently observed natural phenomenon. Nature, 224, 1102-1103.

Jackson, R. D., Clarke, T. R., \& Moran, S. (1992). Bidirectional calibration results for 11 spectralon and $16 \mathrm{BaSO}_{4}$ reference panels. Remote Sensing of Environment, 40, 231-239.

Jacquemoud, S., Verhoef, W., Baret, F., Bacour, C., Zarco-Tejada, P. J., Asner, G. P., et al. (2009). PROSPECT + SAIL models: a review of use for vegetation characterization. Remote Sensing of Environment, 113, S56-S66.

Levizou, E., Drilias, P., Psaras, G. K., \& Manetas, Y. (2004). Nondestructive assessment of leaf chemistry and physiology through spectral reflectance measurements may be misleading when changes in trichome density co-occur. New Phytologist, 165, 463-472.

Lewis, P. (2007). 3D canopy modelling as a tool in remote-sensing research. Dordrecht: Springer.

Li, X., \& Strahler, A. H. (1985). Geometric-Optical Modeling of a Conifer Forest Canopy. IEEE Transactions on Geoscience and Remote Sensing, GE-23, 705--721.

Markwell, J., Osterman, J. C., \& Mitchell, J. C. (1995). Calibration of the Minolta SPAD502 leaf chlorophyll meter. Photosynthesis Research, 46, 467-472.

McClendon, J. H., \& Fukshansky, L. (1990). On the interpretation of absorption spectra of leaves-1. Introduction and the correction of leaf spectra for surface reflection. Photochemistry and Photobiology, 51, 203-210.

Mistele, B., \& Schmidhalter, U. (2008). Estimating the nitrogen nutrition index using spectral canopy reflectance measurements. European Journal of Agronomy, 29, 184.

Moreau, O., Curt, J. N., \& Leroux, T. (2000). Contrast and colorimetry measurements versus viewing angle for microdisplays. Proceedings of SPIE, 4207, 20-30.

Nelder, J. A., \& Mead, R. A. (1965). A simplex method for function optimization. The Computer Journal, 7, 308-313.

Obein, G., Knoblauch, K., \& Viénot, F. (2004). Difference scaling of gloss: Nonlinearity, binocularity, and constancy. Journal of Vision, 4

Obein, G., Leroux, T., \& Vienot, F. (2001). Bi-directional reflectance distribution factor and gloss scales. In B. E. P. T. N. Rogowitz (Ed.), Human Vision and Electronic Imaging $V i$ (pp. 279-290).

Rondeaux, G., \& Vanderbilt, V. C. (1993). Specularly modified vegetation indices to estimate photosynthetic activity. International Journal of Remote Sensing, 14, 1815-1823.

Roujean, J. L., Leroy, M., \& Deschamps, P. Y. (1992). A bidirectional reflectance model of the Earth's surface for the correction of remote sensing data. Journal of Geophysical Research, 97, 20455-20468.

Rykowski, R. a. L., J. (2008). Novel Technology for view angle performance measurement (pp. 41-42). : IMID/IDMC.

Saleh, B. E. A., \& Teich, M. C. (1991). Fundamentals of Photonics.

Sanz, C., Espana, M., Baret, F., Weiss, M., Vaillant, L., Hanocq, J. F., et al. (1997). Bi-directional characteristics of leaf reflectance and transmittance: measurement and influence on canopy bi-directional reflectance. In G. Guyot, \& T. Phulpin (Eds.), 7th International Symposium on physical measurements and signatures in remote sensing (pp. 583-590). Courchevel (France): Balkema.

Sarto, A. W., Woldemar, C. M., \& Vanderbilt, V. C. (1989). POlarized Light Angle instrument I polarized incidence (POLAR:I). In SPIE (Ed.), San Diego, California, (USA: SPIE.

Saunderson, J. L. (1942). Calculation of the color of pigmented plastics. Journal of the Optical Society of America, 32, 727-729.

Slaton, M. R., Hunt, E. R. J., \& Smith, W. K. (2001). Estimating near-infrared leaf reflectance from leaf structural characteristics. American Journal of Botany, 88, 278-284.

Stuckens, J., Somers, B., Delalieux, S., Verstraeten, W. W., \& Coppin, P. (2009). The impact of common assumptions on canopy radiative transfer simulations: a case study in Citrus orchards. Journal of Quantitative Spectroscopy and Radiative Transfer, $110,1-21$

Terashima, I., \& Saeki, T. (1983). Light environment within a leaf I. Optical properties of paradermal sections of Camellia leaves with special reference to differences in the optical properties of palissade and spongy tissues. Plant $\mathcal{E}$ Cell Physiology, 24, 1493-1501.

Torrance, K. E., \& Sparrow, E. M. (1967). Theory for off-specular reflection from roughened surfaces. Journal of the Optical Society of America, 57, 1105-1112.

Walter-Shea, E. A., Norman, J. M., \& Blad, B. L. (1989). Leaf bidirectional reflectance and transmittance in corn and soybean. Remote Sensing of Environment, 29, 161-174. 